\title{
Acute-phase effects of single-time topical or systemic corticosteroid application immediately after hot water-induced burn injury of various grades
}

\author{
Naotaka Doi ${ }^{*}$, Yumi Nakatani, Yutaka Inaba ${ }^{1}$, \\ Toshikazu Kondo ${ }^{2}$, Fukumi Furukawa ${ }^{1}$, Nobuo Kanazawa ${ }^{1}$ \\ ${ }^{1}$ Department of Dermatology, Wakayama Medical University, Wakayama, Japan \\ ${ }^{2}$ Department of Forensic Medicine, Wakayama Medical University, Wakayama, Japan
}

\section{ABSTRACT}

The aim of this study is to evaluate the effects of corticosteroid application on each grade of burn, and to clarify the underlying mechanisms of the effects, especially in its acute inflammatory phase. To generate three-graded burn models (epidermal burn, or EB; dermal burn, or DB; and subcutaneous burn, or SB), hot water was applied on the back skin of Hos:HR-1 mice. Strongest-class (or high-potent) corticosteroid ointment (DD group) or petrolatum (control group) was applied on the back immediately after the hot water application on mice. Prednisolone sodium succinate (PDN group), $1 \mathrm{mg} / \mathrm{kg}$ was orally applied immediately after the hot water application on mice. The mice were sacrificed 1-3 days after hot water application, and the lesional skin samples were provided for histological assessment to enumerate the number of infiltrating inflammatory cells. The mRNA expression levels of inflammatory cytokines $(I L-1 \beta, T N F \alpha, I L-6$ and $I F N \gamma)$ in the lesional skin were also investigated. As a result, corticosteroid application suppressed the number of infiltrating inflammatory cells in the DD group of EB and SB at the early phase, and in DB at all timepoints. However, the number of infiltrating inflammatory cells increased in EB on day 3. Expression of cytokines was generally suppressed in the PDN group of SB. In the cases of EB and DB, some cytokines had decreased but many of the others showed increased expression. In conclusion, the anti-inflammatory effects of corticosteroids are not simple inhibitory effects on inflammatory cell infiltration and cytokine production, but exert more complicated effects in vivo.

Keywords: burn; corticosteroid; inflammatory cell infiltration; cytokine; mouse model

ARTICLE INFO

Received: January 15, 2019

Accepted: February 16, 2019

Available online: March 2, 2019

*CORRESPONDING AUTHOR

Naotaka Doi, Department of Dermatology,

Wakayama Medical University, 811-1

Kimiidera, Wakayama 641-0012, Japan;

doiod@wakayama-med.ac.jp

CITATION

Doi N, Nakatani Y, Inaba Y, et al. Acute-

phase effects of single-time topical

or systemic corticosteroid application

immediately after hot water-induced

burn injury of various grades. Trends

Immunother 2019; 3(1): 10-18.

doi: 10.24294/ti.v3.i1.14

\section{COPYRIGHT}

Copyright (C) 2019 by author(s) and EnPress Publisher LLC. This work is licensed under the Creative Commons AttributionNonCommercial 4.0 International License (CC BY-NC 4.0). http://creativecommons. org/licenses/by/4.0/

\section{Introduction}

Burns are one of the most common and devastating forms of trauma. For clinical applications, corticosteroid ointment is widely used for the initial care of a burn. The suppression of inflammation and accompanying pain and tissue damage is considered to be the theoretical basis of this application. However, no clear evidence has ever been reported. The healing of a skin wound is a systemic process consisting of three sequential phases including inflammation, proliferation and maturation. These phases are progressed by a complicated interaction of cells of various types ${ }^{[1]}$. A number of studies have demonstrated that a variety of cytokines, chemokines, growth factors and proteases are intimately involved in this process ${ }^{[1,2]}$. Therefore, it would provide useful information to clarify any alteration of lesional inflammatory cell infiltration and cytokine production in the inflammatory phase after corticosteroid application.

The aim of this study is to prepare mouse models of various grades of burn and, using these models, to evaluate the effects of corticosteroid application on each grade of burn in the acute inflammatory phase, especially on lesional infiltration of inflammatory cells and production of inflammatory cytokines. 


\section{Materials and Methods}

\section{Generation of mouse burn models}

Hairless Hos:HR-1 mice were purchased from SLC (Hamamatsu, Japan). To generate threegraded burn models, hot water was applied with a cotton sponge on the back skin of adult ( 8 to 12 weeks old) female Hos:HR-1 mice ${ }^{[3]}$. To establish a model of epidermal burn (EB), dermal burn (DB) and subcutaneous burn (SB), hot water was applied at $70{ }^{\circ} \mathrm{C}$ for $1 \mathrm{~s}$, at $80{ }^{\circ} \mathrm{C}$ for $5 \mathrm{~s}$ and at 80 ${ }^{\circ} \mathrm{C}$ for $10 \mathrm{~s}$, respectively. To examine the effects of topical corticosteroid application, a strongest-class corticosteroid ointment (diflorasone diacetate, or DD) was applied on the back immediately after the hot water application in some mice. As a control, petrolatum was applied in some other mice. To examine the effects of systemic corticosteroid application, $1 \mathrm{mg} / \mathrm{kg}$ of prednisolone sodium succinate (PDN) was fed immediately after the hot water application in other mice $(n=10$ in each group). All mice were fed with standard feed and given water ad libitum. This research protocol was approved by the Animals Care Committee of Wakayama Medical University.

\section{Histological and immunohistochemical analyses}

The mice were sacrificed 1-3 days after the hot water application, and the lesional skin samples were isolated and paraffin-embedded sections were made, followed by hematoxylin and eosin (HE) staining. In another series, some of them were immunostained with rabbit anti-mouse myeloperoxidase (MPO) polyclonal antibodies (pAb) (NeoMarkers, Fremont, $\mathrm{CA}$ ) and rabbit anti-human CD3 pAbs (Dako Japan, Tokyo, Japan). The number of infiltrating inflammatory cells was enumerated using WinRoof ${ }^{B}$ software (Mitani Corporation, Tokyo, Japan).

\section{Quantitative analysis of local cytokine expression}

The mRNA expression levels of inflammatory cytokines $(I L-1 \beta, I L-6, T N F \alpha$ and $I F N \gamma)$ in the lesional skin were investigated by real-time polymerase chain reaction (PCR) using SYBR Green Real-Time PCR System (Roche Diagnostics, Basel, Switzerland). $\beta$-actin was used for internal control. PCR primers were purchased from Nihon Gene Research Laboratories Inc. (Sendai, Japan). Each experiment was repeated three times and the mean \pm standard errors of the mean (SEM) was shown.

\section{Statistical analyses}

Statistical analyses were performed with the Student's $t$-test.

\section{Results}

\section{Generation of hot water-induced three grades of burn model on hairless mice}

According to the protocol approved by the Animals Care Committee of Wakayama Medical University, hot water was applied on the back of hairless Hos:HR-1 mice to generate three grades of burn model. Hot water application at $70{ }^{\circ} \mathrm{C}$ for 1 s caused only EB, while DB or SB was stably developed when hot water was applied at $80^{\circ} \mathrm{C}$ for 5 $\mathrm{s}$ or at $80^{\circ} \mathrm{C}$ for $10 \mathrm{~s}$, respectively (Figure 1).

\section{Variable effects of corticosteroid application on burn grade-dependent inflammatory cell infiltration}

On the histological analysis, the change in the number of infiltrating inflammatory cells was compared between control, DD and PDN groups. In general, the deeper the burn, the higher the number of infiltrating inflammatory cells were observed (EB $<\mathrm{DB}<\mathrm{SB}$ ), as shown in Figure 2. Since the ratio of the sum of MPO-positive and CD3-positive cells
(A)

(B)

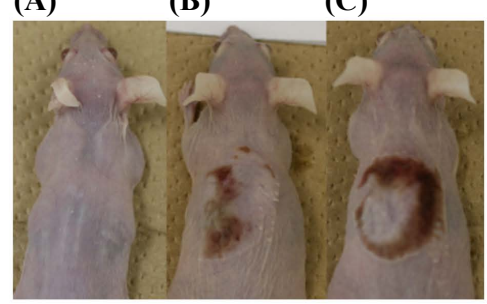

(D)

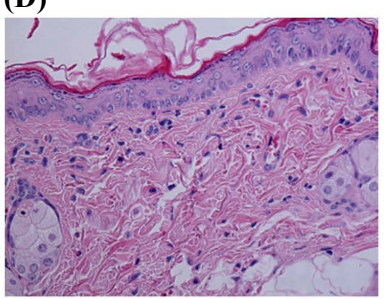

(E)

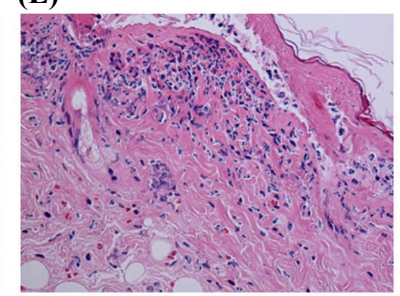

(F)

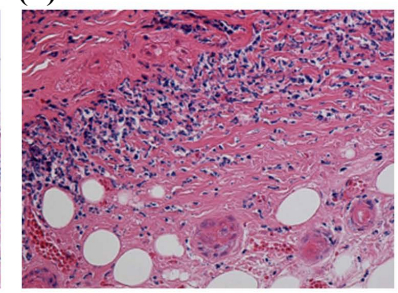

Figure 1. Gross and histological pictures of each degree of burn. Hot water was applied on hairless back skin of Hos:HR-1 mice to induce epidermal burn, or EB (A); dermal burn, or DB (B); and subcutaneous burn, or SB (C). Original magnification $200 \times$ of EB (D), DB (E), SB (F). 
(A)

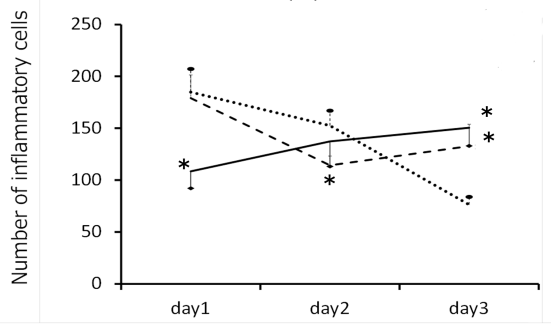

(B)

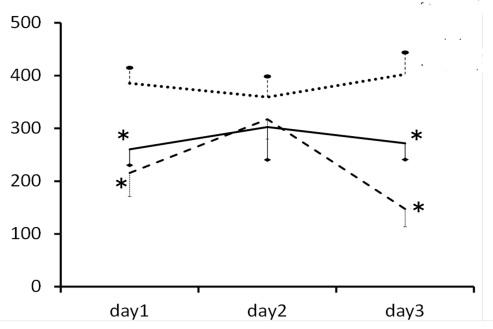

$\cdots$ control

$-D D$

$--P D N$

Figure 2. The number of infiltrating inflammatory cells in each degree of burn with/without corticosteroid application. After hot water-induced burn injury, lesional skin was isolated at indicated time-points and provided for hematoxylin and eosin staining. The dotted line, solid line and dashed line indicate control, corticosteroid (DD) and Prednisolone sodium succinate (PDN) groups, respectively. Statistical analyses were performed against the control group on each day. ${ }^{*} p<0.05$, (A) epidermal burn, or EB, (B) dermal burn, or DB, (C) subcutaneous burn, or SB. Bars indicate standard errors of the mean (SEM).

in all infiltrating cells measured by HE stain reached almost $100 \%$ in EB, $95 \%$ in DB or $89 \%$ in SB (data not shown), most of the infiltrating inflammatory cells were neutrophils and lymphocytes.

In the case of EB, the number of infiltrating inflammatory cells was highest on day 1 and decreased for another two days in control mice. However, in the DD group, it was significantly lower on day 1 than in control and then increased for another two days to become significantly higher than the control on day 3 (Figure 2A). On the other hand, in the PDN group, it was almost the same level on day 1 but decreased to a significantly lower level on day 2 compared with the control, and then increased to a similar level as the DD group on day 3. These results suggest that inflammatory cell infiltration induced by EB was immediate and temporal (highest on or before day 1) event and topical corticosteroid application induced delayed and slower but sustained infiltration of inflammatory cells, while oral corticosteroid application showed delayed and only temporal inhibition of inflammatory cell infiltration.

In the case of $\mathrm{DB}$, the number of infiltrating inflammatory cells in control mice reached almost twice that of EB on day 1 and remained at a similar level for another two days (Figure 2B). In the DD group, it was significantly lower than the control on day 1 and for another two days. In the PDN group, it was further decreased on days 1 and 3. These results suggest that DB-induced inflammatory cell infiltration was a prolonged (more than 3 days) event and topical and oral corticosteroid applications similarly showed stable inhibition of inflammatory cell infiltration.

In the case of SB, the maximum number of infiltrating inflammatory cells in control mice was observed on day 2 and was almost three times more than that of EB (Figure 2C). In the DD group, the number of infiltrating inflammatory cells was significantly lower only on day 1 . In contrast, the number of infiltrating inflammatory cells in the PDN group was highest on day 1, without any significant difference from that in control at any time-point. These results suggest that inflammatory cell infiltration induced by SB was a delayed but temporal (highest on day 2) event and topical corticosteroid application showed immediate and only temporary inhibition of inflammatory cell infiltration, while oral corticosteroid application showed only small effects.

\section{Variable effects of corticosteroid application on burn grade-dependent local inflammatory cytokine production}

By quantitative PCR, the change in the lesional mRNA expression of inflammatory cytokines, including $I L-1 \beta, T N F \alpha, I L-6$ and $I F N \gamma$, was compared between control, DD and PDN groups. In general, higher production of inflammatory cytokines was commonly observed in deeper burns $(\mathrm{EB}<\mathrm{DB}$ $<\mathrm{SB}$ ), as shown in Figure 3.

In the case of EB, weak production of IL-1 $\beta$, TNF $\alpha$ and IFN $\gamma$, but almost no IL-6, was observed with a peak of IL-1 $\beta$ and IFN $\gamma$ on day 2 and TNF $\alpha$ on day 1 in control mice (Figure 3A-D). IL-1 $\beta$ production was significantly lower in the DD group than in control on days 1 and 2, while it was higher on day 3 without significant difference. In the PDN group, it was higher on day 1 without significant difference, and significantly higher on days 2 and 3 (Figure 3A). TNF $\alpha$ production was significantly lower in the DD group than in control from day 1 to 3 , while the same effect was observed only on day 2 in the PDN group (Figure 3C). Weak production of IL-6 was observed in both DD and PDN groups, 
which was higher than in control on days 1 and 2 in the DD group and on day 3 in the PDN group without significant difference (Figure 3B). IFN $\gamma$ production was significantly lower than in control on day 2 in the DD group. In contrast, it was higher than in control on days 1 and 3 in the PDN group without significant difference (Figure 3D).

To summarize, in our model of EB, weak mRNA expression of $T N F \alpha$ with following $I L-1 \beta$ and $I F N \gamma$ was transiently induced. Most of the inflammatory cytokine production was significantly inhibited by topical corticosteroid, whereas significant upregulation of IL-1 $\beta$ production on days 2 and 3 and the same tendency of other cytokines, except for inhibition of TNF $\alpha$ production on day 2, was observed after oral corticosteroid application.

In the case of $\mathrm{DB}$, almost ten times more $I L-1 \beta$ and two times more $I L-6$ and $I F N \gamma$ mRNA expression was observed than in EB (Figure 3A-H). In control mice, mRNA expression of all four cytokines was only limited at all time-points, with an exception for much increased $T N F \alpha$ expression on day 3 . IL- $1 \beta$ production was significantly higher than in control, on day 3 in the DD group and on days 2 and 3 in the PDN group (Figure 3E). IL-6 production also tended to be higher than in control on days 2 and 3 in both DD and PDN groups, whereas it was significantly lower on day 1 in the PDN group (Figure 3F). For TNF $\alpha$ and IFN $\gamma$ production, the DD group showed almost the same level as the control at all time-points. In the PDN group, TNF $\alpha$ production gradually declined to a lower level than in control on day 3 without significant difference (Figure 3G). In contrast, IFN $\gamma$ production in the PDN group was significantly higher on days 1 and 2, but had decreased to almost the same level on day 3 , compared with that in control (Figure 3H).

To summarize, in our DB model, mRNA expression of $I L-1 \beta, T N F \alpha, I L-6$ and $I F N \gamma$ was quite limited, and only $T N F \alpha$ was significantly increased on day 3. Topical corticosteroid application showed almost no effect, except for significantly elevated production of IL- $1 \beta$ on day 3 . For oral corticosteroid application, in addition to the similar effects, decreased TNF $\alpha$ production on day 3 and IL- 6 production on day 1 and increased IFN $\gamma$ production on days 1 and 2 were also observed .

In the case of $\mathrm{SB}$, almost two times more IL-1 $\beta$ and IFN $\gamma$ and four or five times more TNF $\alpha$ and IL-6 mRNA expression was observed than in DB (Figure 3E-L). In control mice, production of IL-1 $\beta$, IL-6 and IFN $\gamma$ peaked on day 1 and gradually decreased after that, while TNF $\alpha$ production peaked on day
2 . In the DD group, IL-1 $\beta$ production tended to be lower on day 1 , but higher on days 2 and 3 than in control (Figure 3I). In the PDN group, it was lower than in control for all three days. The same tendency was also observed for IL-6 and IFN $\gamma$ production in the PDN group and for IL-6 production in the DD group (Figures $\mathbf{3 J}$ and $\mathbf{L}$ ). TNF $\alpha$ production was significantly lower on day 2 in both DD and PDN groups, and tended to be lower on day 1 in the DD group and on day 3 in the PDN group, than in control (Figure 3K). In the PDN group, IFN $\gamma$ production tended to be lower on day 1 than in control, while it gradually increased and tended to be higher on day 3 (Figure 3L).

To summarize, in our SB model, mRNA expression of $I L-1 \beta, I L-6$ and $I F N \gamma$ with following $T N F \alpha$ was transiently induced. Oral corticosteroid application decreased any cytokine production at all time-points, whereas topical corticosteroid application increased IL- $1 \beta$ and IFN $\gamma$ production on days 2 and 3 .

Comparison of the effects of corticosteroid application on burn grade-dependent inflammatory cell infiltration and local cytokine production

To compare the variable effects of corticosteroid application more directly, the ratio of the number of infiltrating inflammatory cells in the DD group to control and that in the PDN group to control, as well as the ratio of relative mRNA expression level in the DD group to control and that in the PDN group to control for each cytokine, were calculated and were summarized in Table 1. Columns including the ratio $<0.5$ and $>1.5$ are highlighted in gray and black, respectively.

In the case of EB, the number of infiltrating inflammatory cells was lower than that of control on days 1 and 2, whereas it showed a greater than 1.5 -fold increase over the control on day 3 in both DD and PDN groups. Similarly, lower expression on days 1 and 2 and higher expression on day 3 of $I L-1 \beta$ and $I F N \gamma$ were observed in the DD group. In contrast, higher expression of $I L-1 \beta$ and $I F N \gamma$ was observed throughout from day 1 to day 3 in the PDN group. $T N F \alpha$ expression was lower in $\mathrm{DD}$, but was almost the same as in the PDN group, compared with control at all time-points. Interestingly, $I L$ 6expression was always higher than control in both DD and PDN groups; it peaked on day 2 in DD and on day 3 in the PDN group.

In the case of $\mathrm{DB}$, the number of inflammatory cells was always lower than control in both DD and 
IL-1 $\beta$

(B)

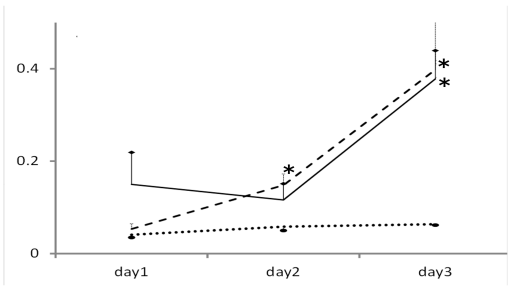

IL-6

(E)

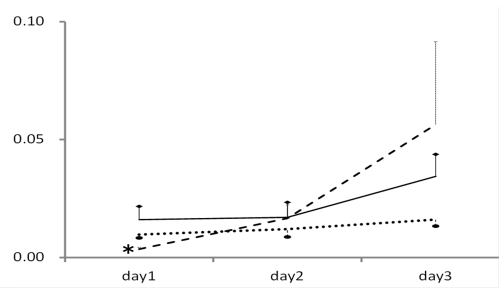

TNF $\alpha$

(H)

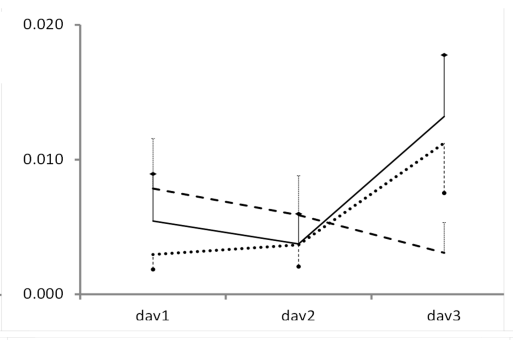

IFN $\gamma$

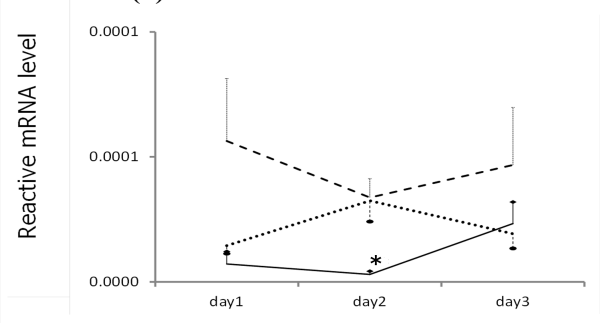

(K)

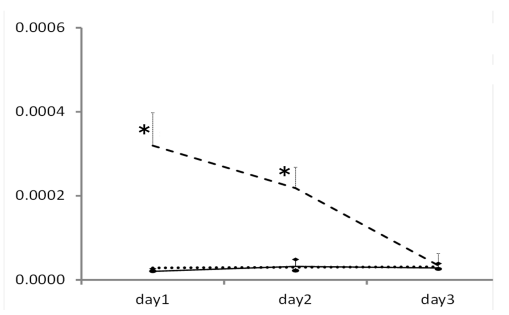

day 1
(G)

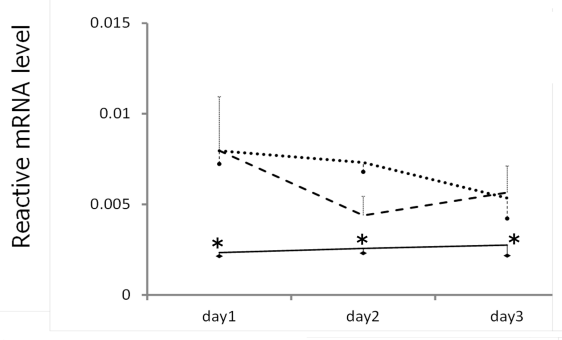

(J)

(D)

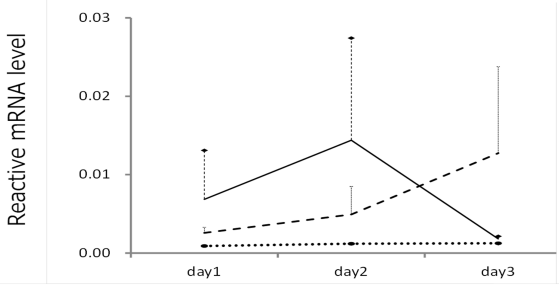

dav
(C)

$\cdots$ control
$-\mathrm{DD}$

-DD

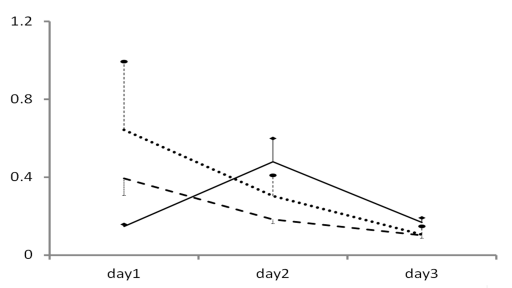

(F)

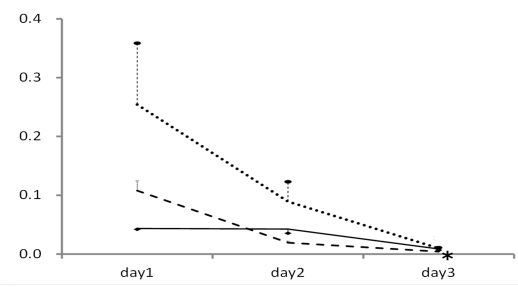

(I)

$\cdots$ control
-DD

-DD

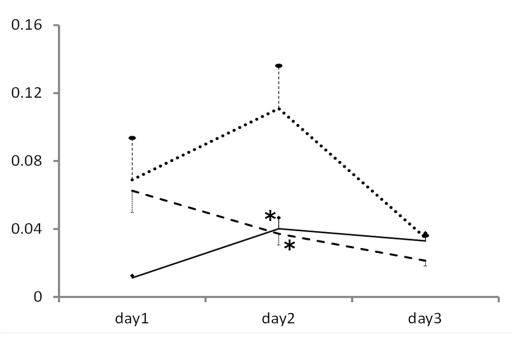

(L)

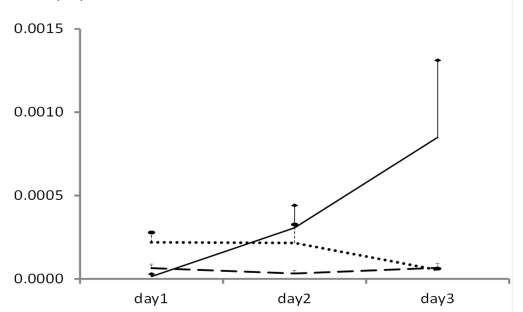

Figure 3. Local mRNA expression of inflammatory cytokines in each degree of burn with/without corticosteroid application. After hot water-induced burn injury, lesional skin was isolated at indicated time-points and provided for real-time PCR analysis. Relative mRNA expression levels were calculated using $\beta$-actin for internal control. The dotted line, solid line and dashed line indicate control, corticosteroid (DD) and Prednisolone sodium succinate (PDN) groups, respectively. ${ }^{*} p<0.05$, (A) $I L-1 \beta$ on epidermal burn, or EB, (B) $I L-1 \beta$ on dermal burn, or $\mathrm{DB},(\mathbf{C}) I L-1 \beta$ on subcutaneous burn, or $\mathrm{SB}$, (D) $T N F \alpha$ on $\mathrm{EB},(\mathbf{E}) T N F \alpha$ on $\mathrm{DB},(\mathbf{F}) T N F \alpha$ on $\mathrm{SB},(\mathbf{G})$ $I L-6$ on EB, (H) IL-6 on DB, (I) IL-6 on SB, (J)IFNy on EB, (K)IFNy on DB, (L)IFNy on SB. Bars indicate standard errors of the mean (SEM). 
Table 1. The ratio of DD/control and PDN/control in the number of infiltrating inflammatory cells and relative mRNA expression levels of inflammatory cytokines in each degree of burn. The ratio of the number of infiltrating inflammatory cells in DD group to control and that in PDN group to control, as well as the ratio of relative mRNA expression level in DD group to control and that in PDN group to control for each cytokine, were calculated and summarized. Columns including the ratio $<0.5$ and $>1.5$ were highlighted in gray and black, respectively.

\begin{tabular}{|c|c|c|c|c|c|c|c|c|c|c|c|c|c|c|c|c|}
\hline & \multicolumn{3}{|c|}{ Cells } & \multicolumn{3}{|c|}{$I L-1 \beta$} & \multicolumn{3}{|c|}{$T N F \alpha$} & \multicolumn{3}{|c|}{$I L-6$} & \multicolumn{3}{|c|}{$I F N \gamma$} \\
\hline & & \begin{tabular}{|c} 
Day \\
1 \\
\end{tabular} & \begin{tabular}{|c} 
Day \\
$\mathbf{2}$ \\
\end{tabular} & \begin{tabular}{|c|} 
Day \\
3
\end{tabular} & $\begin{array}{c}\text { Day } \\
1\end{array}$ & \begin{tabular}{|c} 
Day \\
2 \\
\end{tabular} & $\begin{array}{c}\text { Day } \\
\mathbf{3}\end{array}$ & $\begin{array}{c}\text { Day } \\
1\end{array}$ & $\begin{array}{c}\text { Day } \\
2\end{array}$ & $\begin{array}{c}\text { Day } \\
3 \\
\end{array}$ & \begin{tabular}{|c|} 
Day \\
1
\end{tabular} & \begin{tabular}{|c|} 
Day \\
2
\end{tabular} & \begin{tabular}{|c} 
Day \\
$\mathbf{3}$ \\
\end{tabular} & \begin{tabular}{|c} 
Day \\
1 \\
\end{tabular} & \begin{tabular}{|c|} 
Day \\
2
\end{tabular} & \begin{tabular}{|c} 
Day \\
3 \\
\end{tabular} \\
\hline \multirow{2}{*}{ EB } & DD & 0.59 & 0.90 & 1.97 & 0.17 & 0.64 & 2.91 & 0.29 & 0.35 & 0.51 & 7.59 & 12.09 & 1.45 & 0.49 & 0.09 & 1.21 \\
\hline & PDN & 0.97 & 0.75 & 1.74 & 2.80 & 1.95 & 3.03 & 1.00 & 0.60 & 1.06 & 2.84 & 4.14 & 10.22 & 3.89 & 1.04 & 2.43 \\
\hline \multirow{2}{*}{ DB } & DD & 0.68 & 0.84 & 0.68 & 3.67 & 1.99 & 5.95 & 1.84 & 1.02 & 1.17 & 1.65 & 1.42 & 2.14 & 0.71 & 1.07 & 0.91 \\
\hline & PDN & 0.56 & 0.88 & 0.37 & 1.30 & 2.55 & 6.26 & 2.65 & 1.60 & 0.27 & 0.36 & 1.38 & 3.51 & 11.28 & 7.27 & 1.11 \\
\hline \multirow{2}{*}{ SB } & DD & 0.47 & 0.78 & 0.93 & 0.23 & 1.58 & 1.59 & 0.16 & 0.36 & 0.95 & 0.17 & 0.48 & 0.88 & 0.07 & 1.42 & 15.93 \\
\hline & PDN & 1.30 & 0.89 & 1.06 & 0.61 & 0.60 & 0.96 & 0.91 & 0.33 & 0.61 & 0.42 & 0.22 & 0.42 & 0.29 & 0.15 & 1.24 \\
\hline
\end{tabular}

Note: EB: epidermal burn; DB: dermal burn; SB: subcutaneous burn; DD: corticosteroid; PDN: Prednisolone sodium succinate

PDN groups. In particular, it was lowest at 0.37 times in PDN on day 3. In contrast, significantly lower expression of cytokines was barely observed. Indeed, lower expression was observed only in cases with $I L-6$ on day 1 and $T N F \alpha$ on day 3 in the PDN group.

In the case of $\mathrm{SB}$, the number of inflammatory cells was lowest on day 1 and increased to be closer to the control on day 3 in the DD group. In the PDN group, it was higher than control on day 1, declined on day 2 and got closer to the control on day 3 . In contrast, significantly higher expression of cytokines was barely observed. Indeed, higher expression was observed only in cases with $I L-1 \beta$ on days 2 and 3 and $I F N \gamma$ on day 3 in the DD group.

In summary, applying corticosteroids for EB suppressed the number of infiltrating inflammatory cells at the early phase, but then markedly increased on day 3. The same tendency was observed for the expression of inflammatory cytokines, such as $I L$ $1 \beta$ and $I F N \gamma$, in the DD group, whereas in the PDN group most cytokines were expressed at higher levels than in control at all time-points. On the other hand, in the case of DB, coincidence of the suppression of inflammatory cell infiltration and the upregulation of cytokine expression was observed at all time-points, with only a few exceptions of the suppressed expression of $I L-6$ on day 1 and $T N F \alpha$ on day 3 in the PDN group. Finally, in the case of SB, the number of inflammatory cells was suppressed in DD, but increased in the PDN group, at the early phase and then got closer to the control on day 3 in both groups. Although expression of cytokines was generally suppressed in the PDN group, higher expression of $I L-1 \beta$ on days 2 and 3 , and much higher expression of $I F N \gamma$ on day 3, were noted in the DD group.

\section{Discussion}

It has long been discussed, without conclusion, of how corticosteroids affect the initial treatment of burns and what kind of influence is exerted by corticosteroids on cytokine production and inflammatory cell infiltration. Furthermore, few data are available on the comparison of systemic corticosteroid application with its topical application for burn injury.

Corticosteroids are well known for their antiinflammatory action. Burn injury causes direct destruction of epidermal and dermal tissue and accompanying increases in inflammatory cell infiltration and cytokine expression, resulting in swelling, pain and fever known as the inflammatory triad. Therefore, it is tempting to believe that suppression of such inflammatory reactions can be achieved by using corticosteroids. Actually, during clinical applications, it is sometimes noticed that corticosteroids seem to improve the burn-induced redness and swelling. Therefore, to clarify the therapeutic effects of corticosteroids on burn injury, we applied corticosteroids locally or systemically on mouse models of hot water-induced various grades of burn and examined the effect of corticosteroids on inflammatory cell infiltration and cytokine expression in their acute inflammatory stage.

On histological analysis, the number of infiltrating inflammatory cells was decreased in the case of the DB model. However, in the case of the EB and SB models, the results were not as expected. In particular, in the case of the EB model, the 
number of inflammatory cells markedly increased on day 3 both in the DD and PDN groups. Since EB only causes transient redness without destruction of epidermal cells, the effect of burn injury was no longer expected on day 3. Although the precise cause of this result is unknown, the fact that a similar effect was observed for the expression of several cytokines in the DD group and, furthermore, most cytokines were always expressed at higher levels than in control in the PDN group, suggest the prolonged inflammation as an adverse effect of corticosteroid application on EB with least skin damage. In the case of the SB model, slightly increased numbers of infiltrating inflammatory cells were observed in the PDN group on day 1 . However, for cytokine expression, no similar effect with rather general suppression by corticosteroid application was observed. Moreover, in spite of the decreased number of infiltrating inflammatory cells in the case of the DB model, increased expression of inflammatory cytokines was observed in most of the same specimens. These results indicate that corticosteroid application on burned skin does not simply decrease inflammatory responses, but rather induces more complicated responses dependent on the depth of burn injury, the method of steroid application and the period after burn.

A subtle spatiotemporal regulation of inflammatory and anti-inflammatory cytokine production is essential for the physiological process of wound healing ${ }^{[4]}$. Serum level of proinflammatory cytokines reflects the systemic response to burn injury and correlates with their local level, which directly reflects the local response to the burn. Proinflammatory cytokines such as IL- $1 \alpha$, IL- $1 \beta$ and $\mathrm{TNF} \alpha$, which belong to the first signaling molecules, were released by damaged keratinocytes and other resident cells in response to epidermal damage ${ }^{[5-7]}$. These cytokines induce the amplification circuit involving various cells. All of the locally produced cytokines contribute to the appearance of fever, production of acute-phase proteins, and an overall status of promoted catabolism. Furthermore, they upregulate the production of IL-6, prostaglandin $\mathrm{E}_{2}$ $\left(\mathrm{PGE}_{2}\right)$ and platelet-activating factor by endothelial cells and macrophages ${ }^{[8,9]}$.

Serum and local levels of IL- 6 are increased after burn injury through its production by fibroblasts, macrophages, endothelial cells and keratinocytes. IL-6 has important functions in the proliferative phase of wound healing. Its downstream effects include indirect induction of neutrophil and macrophage infiltration, collagen deposition, angiogenesis, epidermal cell proliferation and tissue remodeling by the induction of tumor growth factor (TGF)- $\beta 1$ and vascular endothelial growth factor (VEGF) production ${ }^{[10,11]}$. Actually, IL-6deficient mice showed delayed wound healing because of reduced granulation tissue formation, re-epithelialization, angiogenesis, infiltration of macrophages and neutrophils, and matrix remodeling ${ }^{[12-14]}$. In burn patients, serum and local IL-6 levels peak approximately one week after injury, and its high level is reportedly associated with increased rates of morbidity and mortality ${ }^{[15]}$. IL- $1 \beta$ and TNF $\alpha$, as well as IL- 6 , contribute to the following $\mathrm{T}$ cell activation through the production of acute-phase proteins ${ }^{[16]}$.

IFN $\gamma$ is another inflammatory cytokine produced by natural killer (NK) cells and T helper (Th) 1 cells in response to injury and is different from the other three cytokines (IL-1, TNF $\alpha$ and IL-6) ${ }^{[17]}$. IFN $\gamma$ has an important role in macrophage activation and differentiation of CD4 T cells into Th1 cells, with inhibition of their differentiation into Th2 cells ${ }^{[18]}$. Activated macrophages and Th1 cells are important in facilitating a proinflammatory response to injury and, hence, IFN $\gamma$ is a cytokine related to the conversion from the inflammatory phase to proliferative phase of wound healing. A set of opposing cell types and cytokines can characterize an anti-inflammatory response and subsequent immunosuppression following burn injury ${ }^{[19]}$. Under specific circumstances such as after severe injury, inhibitory macrophages produce increased amounts of $\mathrm{PGE}_{2}$ and decreased amounts of IL-12, which has a cooperative effect on Th1 cell differentiation ${ }^{[9,20,21]}$. Accordingly, CD4 T cells begin to differentiate into $\mathrm{Th} 2$ cells, which produce anti-inflammatory cytokines IL- 4 and IL-1 $0^{[18,22]}$. In particular, in the initial phase of burn, increased expression of IL1 , TNF $\alpha$ and IL- 6 is induced in the Th1-dominant state. Subsequently, increased expression of antiinflammatory cytokines is induced in the shifting from the Th1-dominant to the Th2-dominant state.

The imbalance of inflammatory and anti-inflammatory cytokine production can result in unbalanced Th1/Th2 polarity with hyper- or hypoinflammation and, subsequently, in delayed wound healing. Indeed, significantly higher levels of IL-6 were detected in chronic long-lasting wounds than in acute rapidly-healing wounds ${ }^{[13]}$. This result suggests that the prolonged inflammation causes delayed wound healing in chronic wounds.

Takano and colleagues studied the time for $50 \%$ healing of wounds in rats which were given corticosteroid intramuscularly for three weeks pre- 
and post-operatively, and found that the time was significantly shorter in the control group than in the corticosteroid-applied group ${ }^{[23]}$. In addition, lower expression of serum cytokines (IL-1 $\beta$, IL-8, TGF $\beta$ and IFN $\gamma$ ) was observed in the corticosteroid-applied group on day 6 . Therefore, long-term administration of corticosteroids may cause insufficient cytokine production in association with impaired wound healing.

Furthermore, corticosteroids generally decrease $\mathrm{PGE}_{2}$ production and can be considered to have an influence on the secondary immunosuppression. An elevated level of glucocorticoids inhibits the production of IFN $\gamma$ and IL-2, but not IL-4 and IL$10^{[9,24,25]}$. These effects can be another reason why corticosteroids cause a delay in wound healing ${ }^{[18,26]}$.

Therefore, in our experiments, corticosteroid was applied only once immediately after the injury to avoid delayed wound healing. However, for cytokine production, clear anti-inflammatory effects of corticosteroids were observed only partly as expected, as follows: lower levels of IL- $1 \beta$ and IFN $\gamma$ only in the early phase and a continuously lower level of TNF $\alpha$ in the DD group of the EB model; a lower level of IL- 6 only in the initial phase and a lower level of TNF $\alpha$ only in the late phase in the PDN group of the DB model; a lower level of all four cytokines only in the early phase in the DD group of the SB model and continuously lower levels of IL-1 $\beta$, TNF $\alpha$ and IL- 6 and a lower level of IFN $\gamma$ only in the early phase in the PDN group of the SB model. Nevertheless, higher expression of cytokines was noted on most of the other conditions.

\section{Conclusion}

These results indicate that corticosteroids do not simply inhibit inflammatory cell infiltration and cytokine production induced by burn injury even in its acute phase; rather, an alteration of the number of infiltrating inflammatory cells and expression of inflammatory cytokines is differentially determined by a set of burn grade and administration route of corticosteroids. Additionally, since lower inflammatory cytokine levels do not necessarily have better effects on wound healing, further studies are required for each application (DD/PDN group in $\mathrm{EB} / \mathrm{DB} / \mathrm{SB}$ model) to determine the precise mechanisms of the effects which corticosteroids exert on each phase of wound healing and to elucidate appropriate directions for the use of corticosteroids on therapeutics for burn injury.

\section{Acknowledgments}

We sincerely thank Mrs. Yumi Kuninaka at the Department of Forensic Medicine, Wakayama Medical University, for her technical assistance. This work was supported by the 2014 Wakayama Medical Award for Young Researchers.

\section{Conflict of Interest}

The authors declare no potential conflict of interest with respect to the research, authorship, and/ or publication of this article.

\section{References}

1. Singer AJ, Clark RAF. Cutaneous wound healing. N Engl J Med 1999; 341: 738-746. doi: 10.1056/ NEJM199909023411006.

2. Martin P. Wound healing-aiming for perfect skin regeneration. Science 1997; 276(5309): 75-81. doi: 10.1126/science. 276.5309 .75 .

3. Fukano K, Nukatsuka H, Takuma K, et al. 創 傷 被覆材の評価のためのラット II 度熱傷モデ ル (Japanese) [A second degree burn model in rats to evaluate wound dressings]. J J Burn Inj 2001; 27(5): 242-251.

4. Lederer JA, Rodrick ML, Mannick JA. The effect of injury on the adaptive immune response. Shock 1999; 11(3): 153-159. doi: 10.1097/00024382199903000-00001.

5. Wood LC, Elias PM, Calhoun C, et al. Barrier disruption stimulates interleukin-1 alpha expression and release from a pre-formed pool in murine epidermis. J Invest Dermatol 1996; 106(3): 397-403. doi: 10.1111/1523-1747.ep12343392.

6. $\mathrm{Hu} \mathrm{Y}$, Liang $\mathrm{D}, \mathrm{Li} \mathrm{X}$, et al. The role of interleukin-1 in wound biology. Part II: In vivo and human translational studies. Anesth Analg 2010; 111(6): 1534-1542. doi: 10.1213/ANE.0 b013e3181f691eb.

7. Hübner G, Brauchl M, Smola H, et al. Differential regulation of proinflammatory cytokines during wound healing in normal and glucocorticoidtreated mice. Cytokine 1996; 8(7): 548-556. doi: 10.1006/cyto.1996.0074.

8. Chaudry IH, Ayala A. Mechanism of increased susceptibility to infection following hemorrhage. Am J Surg 1993; 165 (2): 59S-67S. doi: 10.1016/S0002$9610(05) 81208-5$.

9. Weissman $\mathrm{C}$. The metabolic response to stress: An overview and update. Anesthesiology 1990; 73: 308-327. doi: 10.1097/00000542-19900800000020 .

10. Abraham E, Freitas AA. Hemorrhage produces abnormalities in lymphocyte function and lymphokine generation. J Immunol 1989; 142(3): 899-906. 
11. Biffl WL, Moore EE, Moore FA, et al. Interleukin- 6 in the injured patient: Marker of injury or mediator of inflammation? Ann Surg 1996; 224(5): 647-664. doi: 10.1097/0000065 8-19961100000009.

12. McFarland-Mancini MM, Funk HM, Paluch $\mathrm{AM}$, et al. Differences in wound healing in mice with deficiency of IL-6 versus IL-6 receptor. J Immunol 2010; 184(12): 7219-7228. doi: 10.4049/ jimmunol.0901929.

13. Lin ZQ, Kondo T, Ishida $\mathrm{Y}$, et al. Essential involvement of IL-6 in the skin wound-healing process as evidenced by delayed wound healing in IL-6-deficient mice. J Leukoc Biol 2003; 73: 713-721. doi: 10.1189/jlb.0802397.

14. Mast BA, Schultz GS. Interactions of cytokines, growth factor, and proteases in acute and chronic wounds. Wound Repair Regen 1996; 4(4): 411420. doi: 10.1046/j.1524-475X.1996.40404.x.

15. Guo Y, Dickerson C, Chrest FJ, et al. Increased levels of circulating interleukin 6 in burn patients. Clin Immunol Immunopathol 1990; 54(3): 361371. doi: 10.1016/0090-1229(90)90050-Z.

16. Xing Z, Gauldie J, Cox G, et al. IL-6 is an antiinflammatory cytokine required for controlling local or systemic acute inflammatory responses. J Clin Investig 1998; 101(2): 311-320. doi: 10.1172/ JCI1368.

17. Schoenborn JR, Wilson CB. Regulation of interferon- $\gamma$ during innate and adaptive immune responses. Adv Immunol 2007; 96: 41-101. doi: 10.1016/S0065-2776(07)96002-2.

18. Gosain A, Gamelli RL. A primer in cytokines. J Burn Care Rehabil 2005; 26(1): 7-12. doi: 10.1097/01.BCR.0000150214.72984.44.
19. Gamelli RL, He L, Liu H. Marrow granulocytemacrophage progenitor cell response to burn injury as modified by endotoxin and indomethacin. J Trauma 1994; 37(3): 339-346. doi: 10.1097/00005373-199409000-00002.

20. Cioffi WG. What's new in burns and meta-bolism. J Am Coll Surg 2001; 92(2): 241-254. doi: 10.1016/S1072-7515(00)00795-X.

21. Göebel A, Kavanagh E, Lyons A, et al. Injury induces deficient interleukin-12 production, but interleukin-12 therapy after injury restores resistance to infection. Ann Surg 2000; 231(2): 253-261. doi: 10.1097/00000658-20000200000015 .

22. DiPiro JT, Howdieshell TR, Goddard JK. Association of interleukin-4 plasma levels with traumatic injury and clinical course. Arch Surg 1995; 130(11): 1159-1162. doi: 10.1001/ archsurg.1995.01430110017004.

23. Takano K, Muto S, Mouri N, et al. The effect of corticosteroids on cytokine levels in the healing wound. Biotherapy 1998; 12(5): 624-626.

24. Faist E, Kupper TS, Baker CC, et al. Depression of cellular immunity after major injury. Its association with posttraumatic complications and its reversal with immunomodulation. Arch Surg 1986; 121(9): 1000-1005. doi: 10.1001/ archsurg.1986.01400090026004.

25. Ramírez F, Fowell DJ, Puklavec M, et al. Glucocorticoids promote a $\mathrm{TH} 2$ cytokine response by $\mathrm{CD}^{+} \mathrm{T}$ cells in vitro. J Immunol 1996; 156 (7): 2406-2412.

26. Wesley AJ. Mechanism of immunologic suppression in burn injury. J Trauma 1990; 30: S70S74. doi: 10.1097/00005373-199012001-00017. 